\title{
Nonunion of the Posteromedial Tubercle of the Talus: Interest of SPECT/CT
}

Delcroix Olivier ${ }^{1}$, Garrigues Florent ${ }^{2}$, Robin Philippe ${ }^{1}$, Abgral Ronan ${ }^{1}$ and Querellou Solène ${ }^{1 *}$

${ }^{1}$ Department of Nuclear Medicine, Brest University Hospital, 2 Avenue FOCH, 26609, Brest Cedex, France

${ }^{2}$ Radiology Department, Cavale Blanche Hospital, boulevard Tanguy-Prigent, 29609 Brest, France

"Corresponding author: Solene Q, Department of Nuclear Medicine, Brest University Hospital, 2 Avenue FOCH, 26609, Brest Cedex, France, Tel: 0033298223327; Email: solene.querellou@chu-brest.fr

Recieved date: June 14, 2016; Accepted date: August 04, 2016; Published date: August 11, 2016

Copyright: (C) 2016 Olivier D, et al. This is an open-access article distributed under the terms of the Creative Commons Attribution License, which permits unrestricted use, distribution, and reproduction in any medium, provided the original author and source are credited.

\begin{abstract}
We report the case of an isolated fracture of the posteromedial tubercle of the talar process caused by a posterior tackle in a football player, which is an unusual and misdiagnosed fracture leading to a nonunion causing a chronic ankle pain. To our knowledge, this is the first reported case demonstrating the major interest of SPECT/CT to diagnose an unusual nonunion of the posteromedial tubercle of the talus.
\end{abstract}

Keywords: Cedell's fracture; SPECT/CT; Posteromedial tubercle; Talus, Nonunion; Scintigraphy

\section{Case Blog}

The below mentioned case of talar fractures are very rare [1] and first case was described by Cedell in 1974 [2]. Rogosic et al. [3] found 26 cases available in 17 reports through PubMed. Misdiagnosing can lead to complications in early phase: there is a risk of interposition of the tendon of the flexor hallucis longus. (FHL $(+)$ ) running in the groove of the posterior process when the bone fragment involved is large, preventing closed reduction [4]. There is also a risk of future subtalar joint arthritis because undersurface of posterior process constitutes around $25 \%$ of the posterior articular facet of the subtalar joint [5]. Moreover, no appropriate treatment goes on to develop painful nonunion for several years [6].
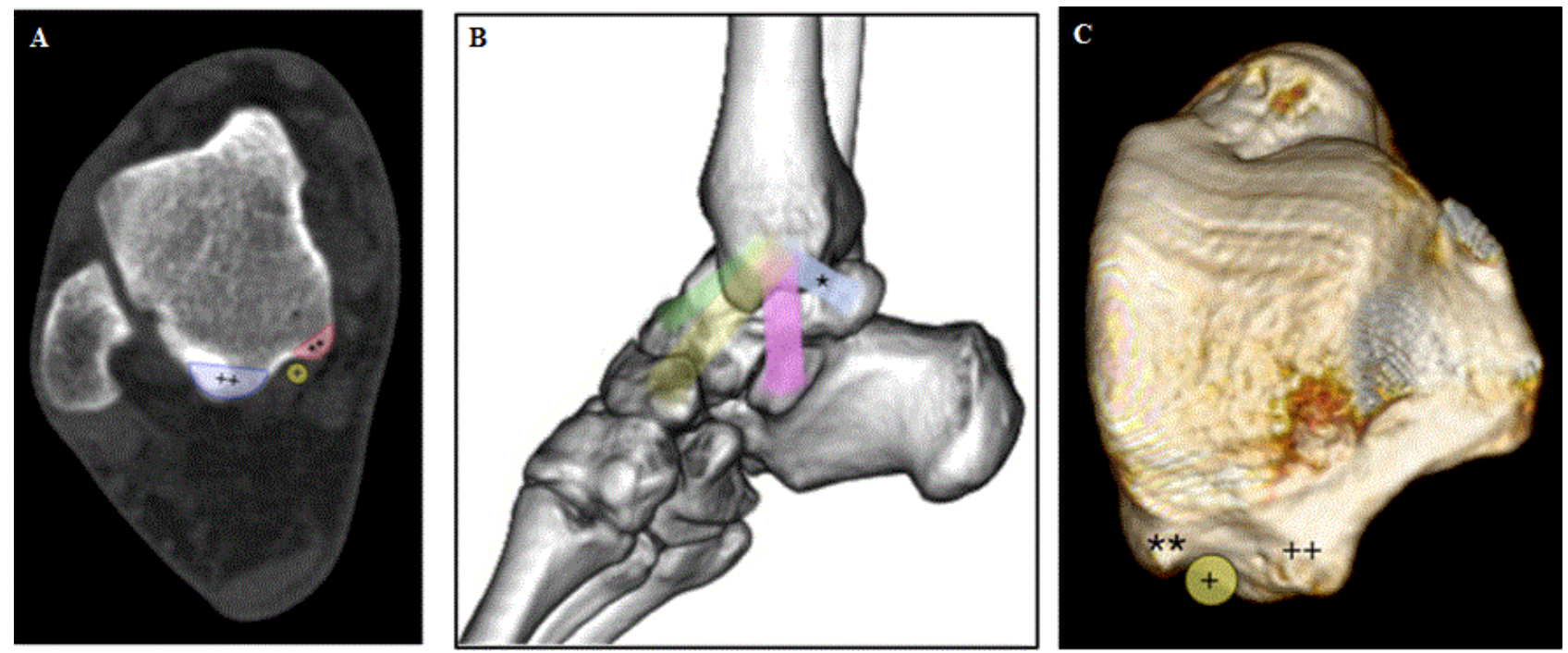

Figure 1: The posterior process of the talus is composed of two tubercles (a): posterolateral tubercle $(++)$ and posteromedial tubercle $\left({ }^{* *}\right)$, where inserts the posterior talotibial ligament $\left.{ }^{*}\right)(\mathrm{b})$ : Talar fractures are unusual and fractures of the medial tubercle are even rarer. (c): (FHL $(+))$ running in the groove of the posterior process when the bone fragment involved is large, preventing closed reduction.

A 18-year-old man was directed to our nuclear department for SPECT/CT 99mTc-MDP imaging to explore a chronic right ankle and rearfoot pain subsequent to a posterior tackle while playing football 13 months earlier. Radiographs performed after trauma did not reveal fracture. Van Tongel et al. [7] had previously described the same fracture in planar bone scan ans Kanbe et al. [8] on computed tomography.
To our knowledge, this is the first reported case demonstrating the major interest of SPECT/CT to diagnose an unusual nonunion of the posteromedial tubercle of the talus. 
Citation: Olivier D, Florent G, Philippe R, Ronan A, Solène Q (2016) Nonunion of the Posteromedial Tubercle of the Talus: Interest of SPECT/CT. J Nucl Med Radiat Ther 7: 299. doi:10.4172/2155-9619.1000299

Page 2 of 3
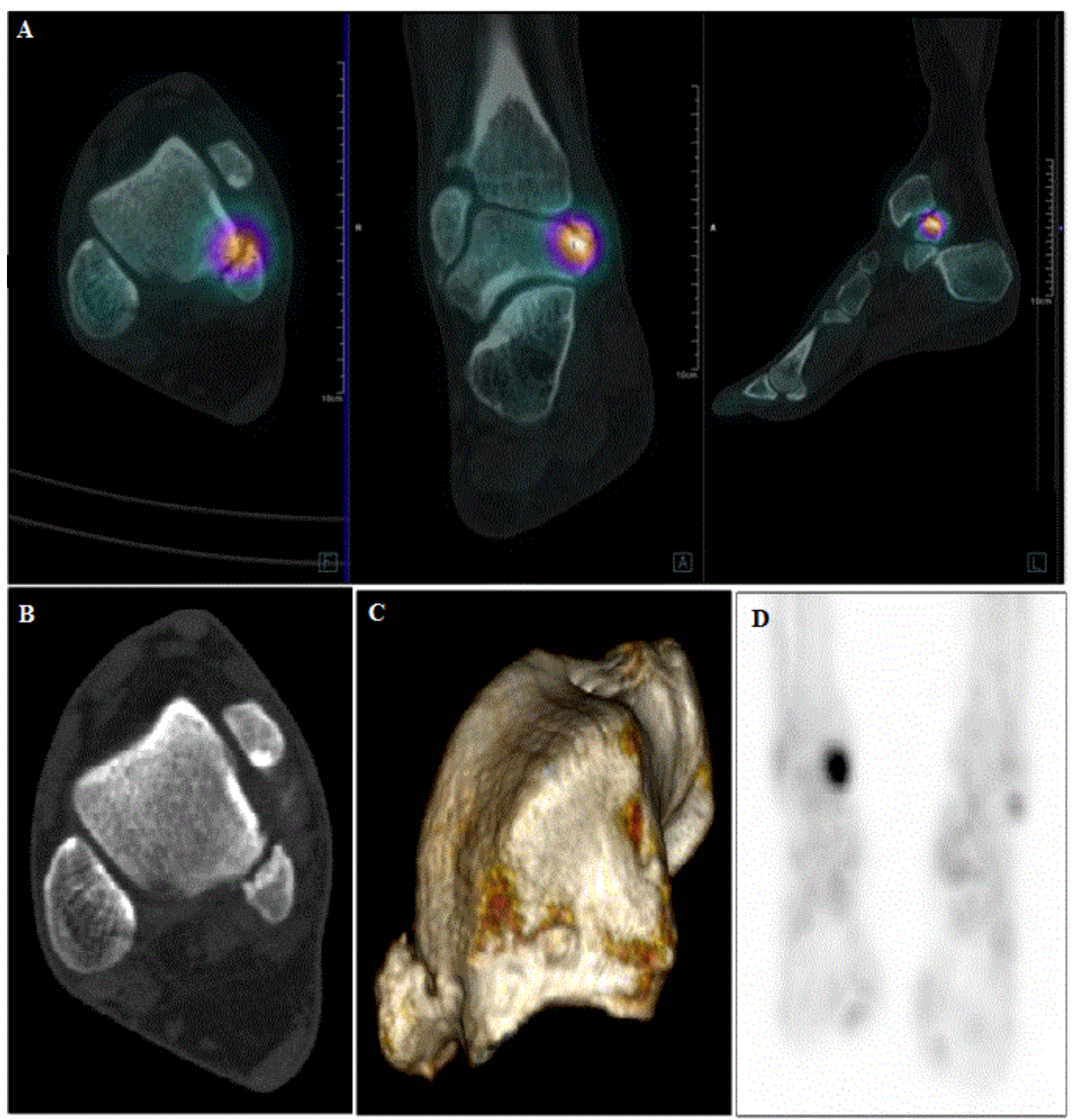

Figure 2: Bone scan showed uptake on an interface (a) with irregular ossification (b) separating the internal side of the posterior process of the talus from a well mineralized nondisplaced hypertrophic bone fragment (c) measuring $15 \times 13 \times 18 \mathrm{~mm}$, revealing hypertrophic nonunion evolution of a fracture of posteromedial tubercle of the talus (d) in SPECT/CT expresses very active bone remodeling, even long after the trauma, reflecting an unfavorable evolution of an unrecognized fracture and explaining the pain.

\section{References}

1. Higgins TF, Baumgaertner MR (1999) Diagnosis and treatment of fractures of the talus: a comprehensive review of the literature. Foot Ankle Int 20: 595-605.
2. Cedell CA (1974) Rupture of the posterior talotibial ligament with the avulsion of a bone fragment from the talus. Acta Orthop Scand 45: 454-461.

3. Rogosic S, Bojanic I, Boric I, Tudor A, Srdoc D, et al. (2010) Unrecognized fracture of the posteromedial process of the talus--a case report and review of literature. Acta Clin Croat 49: 315-320. 
Citation: Olivier D, Florent G, Philippe R, Ronan A, Solène Q (2016) Nonunion of the Posteromedial Tubercle of the Talus: Interest of SPECT/CT. J Nucl Med Radiat Ther 7: 299. doi:10.4172/2155-9619.1000299

Page 3 of 3

4. Dougall TW, Ashcroft GP (1997) Flexor hallucis longus tendon interposition in a fracture of the medial tubercle of the posterior process of the talus. Injury 28: 551-552.

5. Rockwood CA, Green DP, Bucholz RW (1991) Rockwood and Green's fractures in adults: Lippincott Company, USA.

6. Albert P, Patel J, Katz JI, Loria F, Parnell J, et al. (2014) Magnetic resonance imaging, computed tomography, and radiographic correlation of nonunion of the posteromedial tubercle of the talus: a case report. J Foot Ankle Surg 53: 787-790.
7. Van Tongel A, Matricali GA (2007) Fracture of the posterior medial tubercle of the talus: a case report and review of the literature. Acta Orthop Belg 73: 804-806.

8. Kanbe K, Kubota H, Hasegawa A, Udagawa E (1995) Fracture of the posterior medial tubercle of the talus treated by internal fixation: a report of two cases. Foot Ankle Int 16: 164-166. 